\title{
WI-FI Based Indoor Positioning Using Fingerprinting Methods (KNN Algorithm) in Real Environment
}

\author{
Samaneh Amirisoori ${ }^{1}$, Salwani Mohd Daud*2, Noor Azurati Ahmad ${ }^{3}$, \\ Nur Syazarin Natasha Abd Aziz ${ }^{4}$, Nurul Iman Mohd Sa'at ${ }^{5}$ \\ and Nur Qamarina Mohd Noor ${ }^{6}$ \\ ${ }^{1}$ CIBC Canada \\ 2,3,4,5,6 Advanced Informatics School, Universiti Teknologi Malaysia, Kuala \\ Lumpur, Malaysia \\ ${ }^{1}$ sammaneh96@yahoo.com,*22salwani.kl@utm.my, ${ }^{3}$ azurati@utm.my, \\ ${ }^{4}$ syazarinnatasha@gmail.com, ${ }^{5}$ nuruliman.mdsaat@yahoo.com.my, \\ ${ }^{6}$ qmnnur2@live.utm.my
}

\begin{abstract}
Global positioning systems have difficulties in finding positions inside buildings. Hence other techniques and methods are required for indoor localization. Since indoor positioning needs additional indoor infrastructures deployment, convenient techniques using Wi-Fi can be developed. In this research, indoor positioning using Wi-Fi access point is investigated as the main usage of Location Based Service (LBS) applications. We employed fingerprinting methods to increase the accuracy of positioning. The study had been done in real environment in Universiti Teknologi Malaysia (UTM). The models were designed using KNN algorithm for indoor positioning. The fingerprinting dataset contained received signal strength from different numbers of existing Wi-Fi access points in the real environment. Additional features were applied to the model in order to enhance the accuracy. The accuracy rate and mean square error were calculated. Evaluations of models had been done by conducting experiments to compare each model with different features. Analysis suggests that KNN method which achieved 77\% of accuracy with $K=7$ is the most precise model for indoor positioning in this study. By applying signal strength from additional access points, more precise results had been achieved and distance errors had been eliminated.
\end{abstract}

Keywords: Indoor positioning, fingerprinting, Wi-Fi, KNN, global positioning systems

\section{Introduction}

Development of navigation systems need sophisticated methods in order to find the current location of a user or device with an adequate accuracy for a given context. Various technologies and methods are available for different type of application. Regarding specific context, the accuracy of these technologies is different from a few meters to a few centimeters. Since location based services integrate location of a mobile user with other information to provide various types of services to a user, knowledge of user position is an essential part of a location based service. Since, navigation have been an essential requirement of the daily life, smart phones are widely used for the navigation purposes nowadays. A location based service has to be consistent in providing navigation in both indoor and outdoor locations. Although the outdoor location based services are successful in providing consistent navigation to the users, indoor positioning and navigation applications are still improving the consistency factor [1].

Received (February 7, 2017), Review Result (August 15, 2017), Accepted (August 25, 2017) 
Outdoor positioning system can be enhanced by combining the satellite positioning systems such as Global Positioning System (GPS), but for indoors environment since we have buildings limitation such as walls or narrow corridors, positioning has been a challenge these days. Therefore, the need for precise indoor positioning during a short time and at any specific position, is forcing us to use efficient positioning systems [2]. In order to propose a Location Based Service for indoor environment, such as hospitals, shopping malls, car parks and underground, so many indoor positioning methods have been proposed via several technologies. These systems are similar to pedestrian localization, object localization and vicinity marketing inside indoor places. There are many various technologies used in the area of indoor navigation like infrared and wireless network (WLAN). Moreover, lots of other techniques are available for localization system: radio frequency identification or RFID which used in a navigation system in Massachusetts Institute of Technology. Active Badge system is an example of infrared technology to propose a service for positioning the room level. Another technology is ultra-wideband (UWB) that is used in manufacturing industries for providing accurate positioning in Ubisense "s positioning system [3].

Regarding the complexity of the indoor environment, large coverage techniques such as cellular base station for indoor localization technique are unreliable. Thus the indoor positioning solution requires additional indoor infrastructure deployment. Unless cheap and convenient techniques such as Wi-Fi can be developed and comprehensive indoor localization continues to be a challenge [4]. According to Vathsangam et al., [5], one of the suitable and cost effective candidate's techniques is using existing Wireless Received Signal Strength (RSS)-based indoor positioning methods. Wi-Fi location determination consists of two primary methods, signal strength propagation models and fingerprinting techniques.

Indoor positioning system need to provide user requirement for indoor location based service. Indoor positioning system should be accurate and easy to use in smart phones. There are lots of approaches for outdoor localization that uses Global Positioning System (GPS) that provides reliable positioning information. However, the GPS is not suitable for indoor localization because satellite signals are weaken through building structures and accordingly, alternative methods are needed to propose an accurate and reliable indoor navigation system [6].

Wi-Fi based indoor positioning is one of the most popular systems that have lots of pre-installed Wi-Fi access points; however its accuracy is not good enough for indoor LBS. In comparison with outdoor environment, the accuracy of Wi-Fi based technology cannot satisfy the expectation of the indoor users because there are lots of Point of Interests (POIs) and narrow corridors which are hard to distinguish the position. Therefore, the indoor service requires more accurate positioning system than outdoor [7]. These days, accurate, reliable and robust indoor locating protocols are increasingly required by more and more users and applications. A real-time locating system gives the positions of mobile devices, and the position information can be used among many services such as navigation, tracking and monitoring [8]. There are many approaches regarding indoor location based service existed, but most of them need additional physical infrastructure except Wi-Fi methods which are available inside most of the building nowadays.

Although there is considerable amount of research, precise indoor positioning is still a long standing problem. The most common technique for indoor navigation is Wi-Fi based approaches, since GPS is not useful inside buildings. Besides, by using Wi-Fi for indoor positioning there is no need for additional peripheral and almost all smart phones are equipped with Wi-Fi features. Despite the robustness of Wi-Fi localization method, there is a challenge for evaluation Wi-Fi signals because of their variety from time to time. The Wi-Fi signals hardly can get stable hence there is much complexities through indoor environment and it is difficult to get precise and flexible signal from wave propagations 
[9]. There is a need to expand the range of Wi-Fi based positioning methods to be analysed and compared, to achieve better insight on the efficient technologies to be deployed. While the requirement of location based services (LBS) in indoor places increases, there is a need for high accuracy positioning methods, that motivates to the development of fingerprinting based positioning approaches which uses wireless local area network (WLAN). They used single fingerprinting algorithm with the integration of several methods [3].

\section{Aim of the Study}

This research is aiming to develop a Wi-Fi based indoor positioning approach using fingerprinting method for mobile users which has acceptable accuracy. This research will investigate prospect of Received Signal Strength Indication (RSSI) algorithms. Besides, the investigation and analysis among fingerprinting methods in real environment inside Universiti Teknologi Malaysia; also simulation and testing will be done along with the result of designed model. Data collection of Received Signal Strength (RSS) will be measured by existing mobile application on user smart phone, in a selected place in Menara Razak (Systems Engineering Lab in level four). Additionally, this research presents comprehensive details of indoor positioning using location fingerprinting algorithm such as K-Nearest Neighbor (KNN).

\section{Methodology}

According to most of researchers work, a real environment can be a room or any place in a building [10]. Our study area is the Systems Engineering Lab which is located in level four of Menara Razak in Universiti Teknologi of Malaysia which contained furniture and equipment such as tables, chairs and computers. Data was collected by measuring the RSSI values of the signals received from the four points. Each point has dedicated label of a, b, c or d as shown in Figure 1 that illustrates a simple view of our research location area map. Our training data collected from these 4 points with the testing data set wasngathered from both known and unknown positions. We used available Wi-Fi access points in wireless network with IEEE 802.11 standards. Figure 2 shows some images presenting the situation of labeled points in real environment.

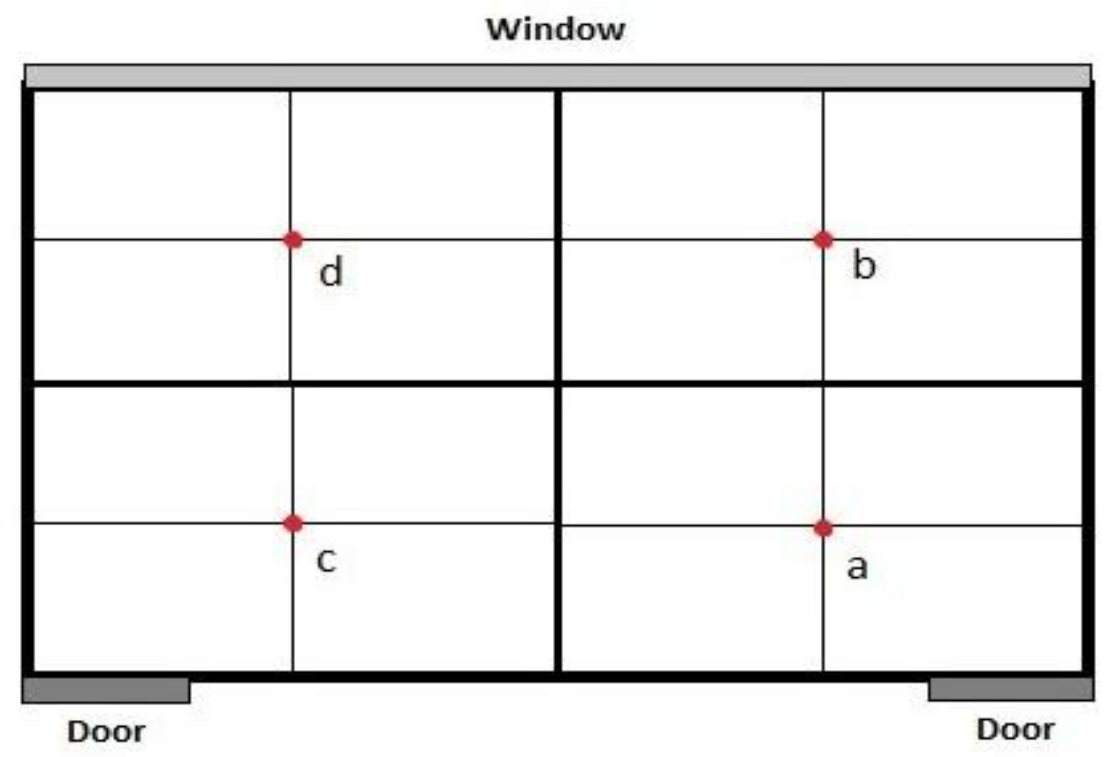

Figure 1. Experiment Layout 


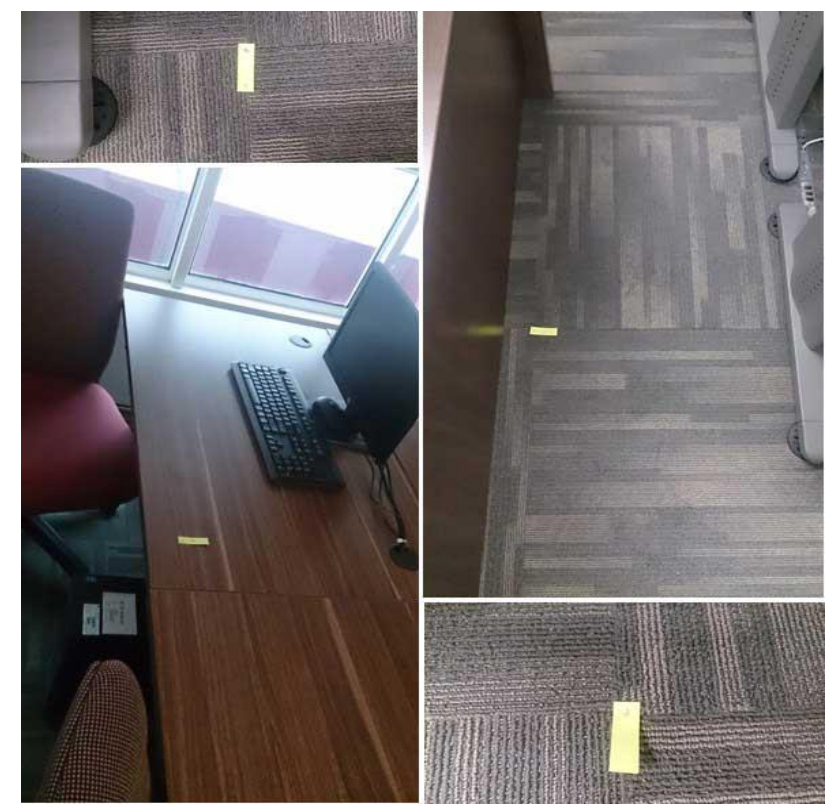

Figure 2. Examples of Labeled Areas

An application called Lightweight Wi-Fi Scanner (APScan) was used to collect signal strengths from Wi-Fi access points. A free and open source application, APScan version 2.3 is $431 \mathrm{kbyte}$ and can be installed on android mobile phones. We used a Sony mobile phone with android OS. This application provides a Comma Separated Value (CSV) file containing information about signal strength that save the date and time of scanning. The structure of information is: BSSID, Power, Channel and ESSID. In this research, we need BSSID which contains access points and Power of the signal. Figure 3 shows an image of running APScan application interface.

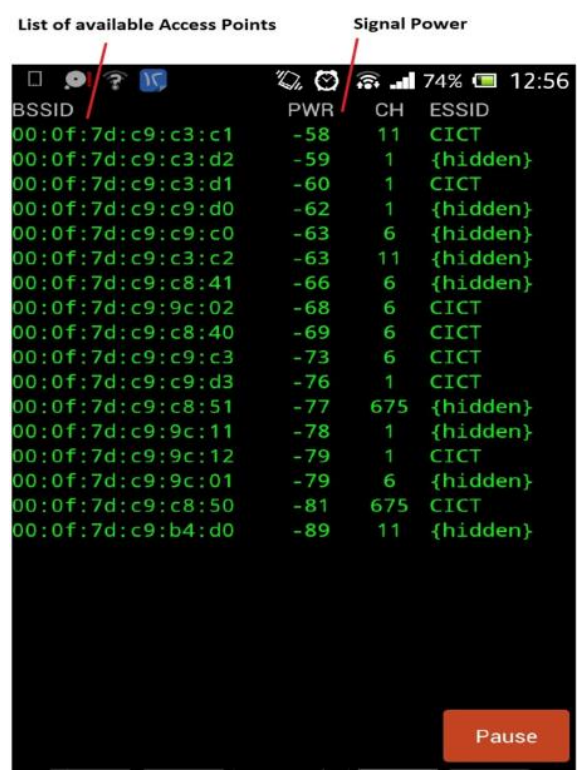

Figure 3. Recording RSS using APScan

\subsection{Data Collection}

Dataset collected in Systems Engineering Lab, which is approximately 8 meters by 5.25 meters, divided into four areas, each point defined in center of four areas which is 
shown by red points in Figure 1. It should be mentioned that the maximum distance between two points is 4 meter. Signal strength measurements done during several weeks and we took the data collection in various day of a week in different days and different times in a day. In each point, we had collected more than 140 samples. The total database contains 560 vectors of RSSI. There were different Media Access Control (MAC) addresses belong to various access points; in this project we considered four to six access points to measure the power. APScan result is a comma separated values (CSV) file containing parameter which is explained in Table 1. There are several BSSID which belongs to every access points. Basically, BSSID is the MAC address which user is not aware of that, when a user moves from one area to another, the MAC address will change because the MAC address belong to another access points but it does not affect the connectivity of wireless network. An example of BSSID is: "08-00-20-ae-fd-7e". The Extended Service Set Identification (ESSID) is the same as BSSI for showing the name of access points. In some wireless network with no access points, the Basic Service Set Identification (BSSID) is used while in some others which network includes an access point, the ESSID is used, but both of them are known as SSID.

Table 1. Values Recorded by APScan

\begin{tabular}{|l|l|}
\hline \multicolumn{1}{|c|}{ Value } & \multicolumn{1}{c|}{ Description } \\
\hline BSSID & $\begin{array}{l}\text { Basic Service Set Identifier is the MAC address of access points } \\
\text { which APScan receives its signal strength. It is unique hexadecimal } \\
\text { number }\end{array}$ \\
\hline Power & Power of signal strength which shows the signal strength length \\
\hline Channel & The channel of the signal \\
\hline ESSID & Extended Service Set Identification, it is the same as BSSID \\
\hline
\end{tabular}

Our study was done in level four, but some BSSIDs collected were associated to access points from other floors. There is a dependency between MAC address and APs. One access point can transfer signals within various MAC addresses. In fact, various different MAC addresses can be associated by one access point. It is needed to classify the MAC addresses which transmit the signal from one access point. In this project, the first 5 characters of the MAC addresses were identical. An example of received signal strength of this project which is the output of the CSV file from APScan is shown in Figure 4. The first row shows BSSID of 00:0f:7d:c9:c3 is the MAC address of one access point like d1, and the power of this access point is -66 .

\begin{tabular}{|c|c|c|c|c|c|}
\hline BSSID & PWR & $\mathrm{CH}$ & ESSID & ENC & DATE \\
\hline & & & & & Scan done on: 6/4/2015 14:35 \\
\hline 00:0f:7d:c9:c3:d1 & -66 & 1 & CICT & Open & \\
\hline 00:0f:7d:c9:c3:c2 & -66 & 11 & $\{$ hidden\} & Open & \\
\hline 00:0f:7d:c9:c3:c1 & -67 & 11 & $\mathrm{CICT}$ & Open & \\
\hline 00:0f:7d:c9:c3:d2 & -67 & 1 & \{hidden\} & Open & \\
\hline 00:0f:7d:c9:c9:d3 & -76 & 1 & CICT & Open & \\
\hline 00:0f:7d:c9:9c:02 & -78 & 6 & CICT & Open & \\
\hline $00: 0 f: 7 d: c 9: c 9: c 0$ & -85 & 6 & \{hidden\} & Open & \\
\hline 00:0f:7d:c9:c9:c3 & -86 & 6 & $\mathrm{CICT}$ & Open & \\
\hline 00:0f:7d:c9:9c:12 & -86 & 1 & CICT & Open & \\
\hline 00:0f:7d:c9:c9:d0 & -86 & 1 & $\{$ hidden\} & Open & \\
\hline 00:0f:7d:aa:1b:33 & -87 & 1 & CICT & Open & \\
\hline 00:0f:7d:aa:1b:31 & -88 & 1 & \{hidden\} & Open & \\
\hline
\end{tabular}

\section{Figure 4. Example of Received Signal Strength File}

\subsection{System Design}

The model of this research which is shown in Figure 5 contains two phases: 
i. Offline phase or training phase includes collecting of RSS samples at reference positions and build a training database. This data base is a distribution of signal strength received from each AP at each point in the location. An example of scattered signal strength power generated from Wi-Fi access points in this research is shown in Figure 6.

ii. Online phase determines the location of a mobile user by comparing the measured RSS values with the training database. In this phase, we developed fingerprinting methods using KNN algorithm. Then the result will be compared and the accuracy of these methods were analyzed.

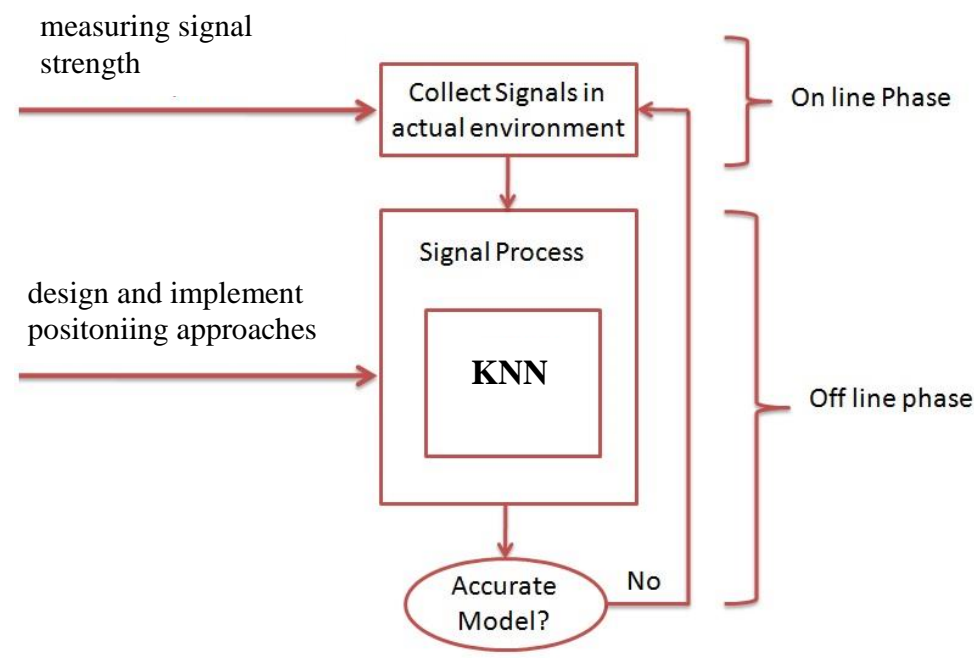

Figure 5. Research Model

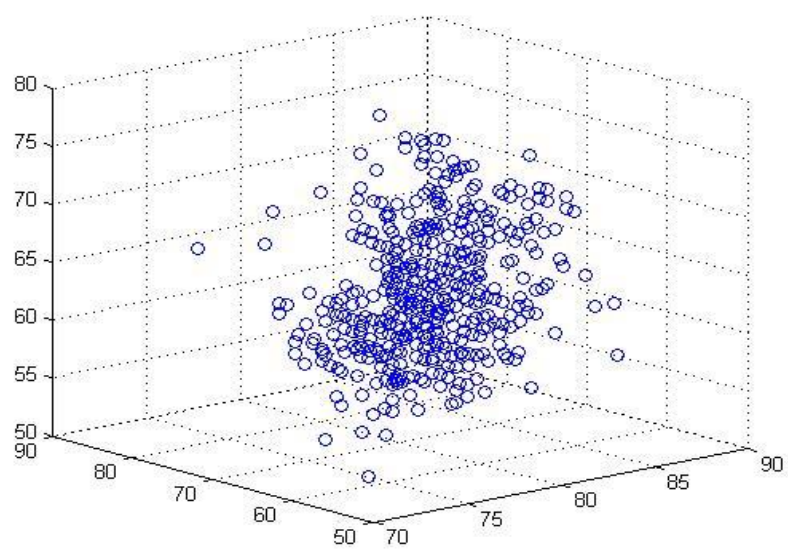

Figure 6. Example of RSS Database in 3D Model

\subsection{K-Nearest Neighbor Method}

We used KNN algorithm based on Euclidean Distance to find the nearest point. The features and steps of this approach are as follows:

- Algorithm: K nearest neighbor using Euclidean Distance

- Goal: Finding the nearest position

- Input: Received Signal Strength from 4 access points (RSS vector)

- Output: Estimated nearest position (can be a, b, c or d) 
- Testing: $70 \%$ training dataset and 30\% testing dataset

Consider our fingerprinting data base: $\left\{\mathrm{RSS}_{\mathrm{Vi}}, \mathrm{RP}_{\mathrm{xi}}\right\}$. The $\mathrm{RP}_{\mathrm{i} . \mathrm{x}}$ shows the location of ith RP, and RSS $\mathrm{Vi}_{\mathrm{i}}$ of ith RP is a vector of $\mathrm{RSS}_{\mathrm{s}}$ which is: $\left\{\mathrm{RSS}_{\mathrm{i} 1}, \mathrm{RSS}_{\mathrm{i} 2}, \ldots, \mathrm{RSS}_{\mathrm{in}}\right\}$. This algorithm simply chooses the fingerprint that has the minimum distance to the current measurement in the signal space. In fact the K nearest neighbors are found by calculating the distance of each RP of database vector from the tag based on their RSSs considering Euclidian distance and the signal strength vector of the mobile user can be \{RSS1, $\mathrm{RSS}_{2} \ldots \mathrm{RSS}_{\mathrm{j}}, \ldots, \mathrm{RSS}_{\mathrm{n}}$, where $\mathrm{RSS}_{\mathrm{j}}$ refers to the RSS of AP jth and can be NULL if that AP is not heard. So, the Euclidian distance of $\mathrm{RSS}_{\mathrm{s}}$ of ith RP and user's is calculated by:

$$
D_{i}=\sqrt{\sum_{j=1}^{n}\left(R S S_{i j}-R S S_{j}\right)^{2}}
$$

Figure 7 illustrates the $\mathrm{K}$ nearest Neighbor algorithm. As it has been shown in this figure for $\mathrm{K}=3$ class $\mathrm{B}$ will be selected as a classification of estimated position.

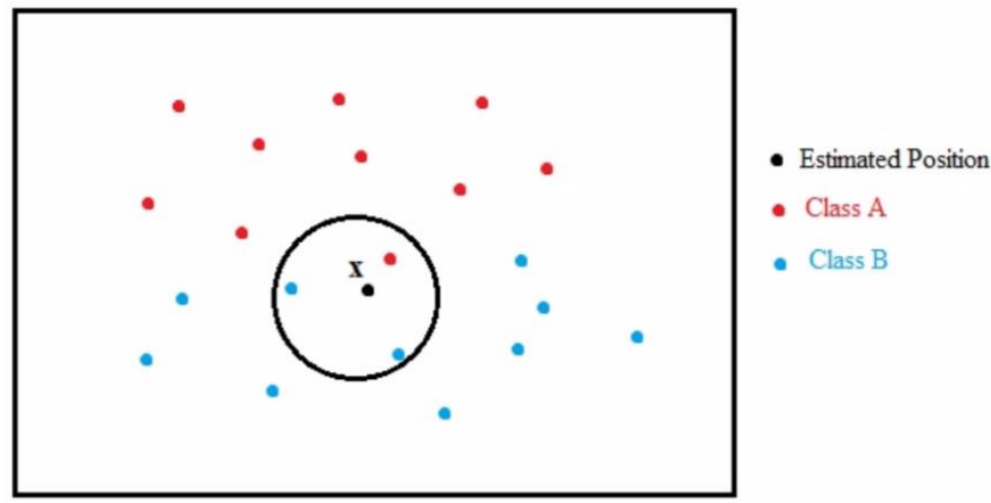

Figure 7. KNN Algorithm

Various $\mathrm{K}$ values were used to test the algorithm and the results obtained were compared. Normally the odd prime numbers will be selected as a $\mathrm{k}$ value, so that we can distinguish the nearest neighbor and to avoid tie situations. The $\mathrm{K}$ value should be a prime number in ideal case to avoid situation like where $\mathrm{K}$ neighbors contains equal number of reference signals from different classes [11]. If the value of $\mathrm{K}$ is smaller then there is higher variance. On the other hand if the value of $\mathrm{K}$ is larger, then there is higher bias, so choosing the proper $\mathrm{K}$ depends on the data. The approach applied and the result for $\mathrm{K}=2$, $3,5,7,11,13,17,19$ was $0.718563,0.706587,0.706587,0.748503,0.724551,0.700599$, 0.682635 and 0.670659 . The result diagram for $\mathrm{K}=2,3,5,7,11,13,17,19$ is shown in Figure 8 . The distance between estimated position and real position in testing dataset was calculated for each value of $\mathrm{K}$, and then the average of error was calculated as a Mean Square Error (MSE) for various value of K. The MSE is calculated by:

$$
M S E=\sqrt{\left(X_{\text {real }}-X_{\text {calc }}\right)^{2}+\left(Y_{\text {real }}-Y_{\text {calc }}\right)^{2}}
$$

This variable shows the average error from testing dataset is measured in meter. The MSE for $\mathrm{K}=2,3,5,7,11,13,17,19$ was $0.985971,1.004175,1.004175,0.839892$, $1.039299,1.063382,1.129381,1.298357$. These values have been rounded and MSE values for various $\mathrm{K}$ are shown in Figure 9. 


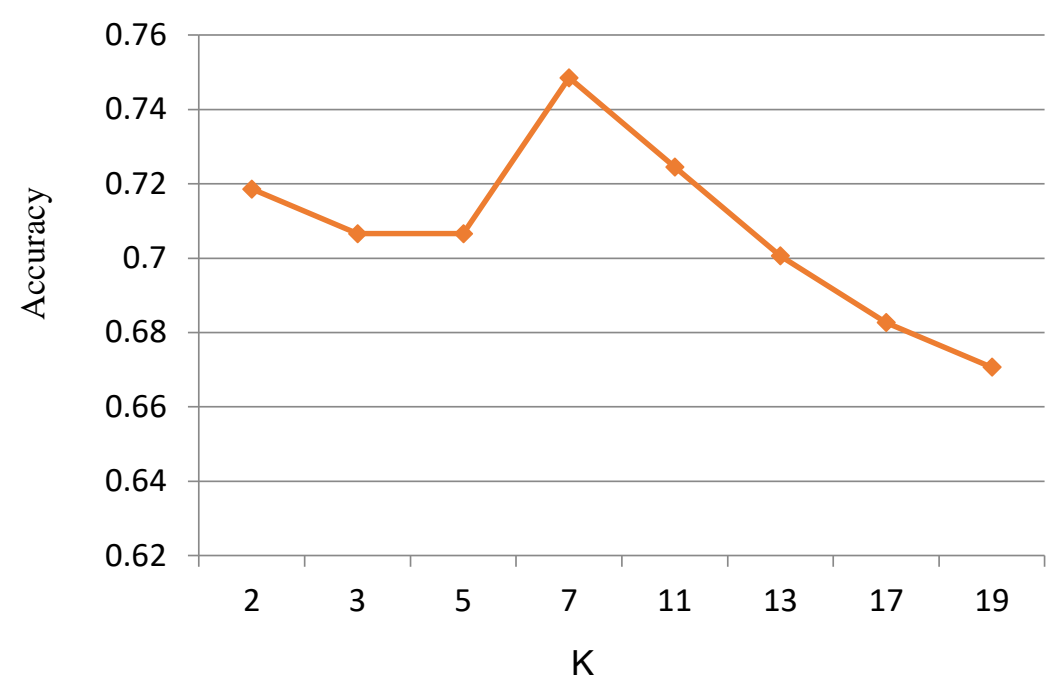

Figure 8. Accuracy Rate for Various Values of K using 4 APs

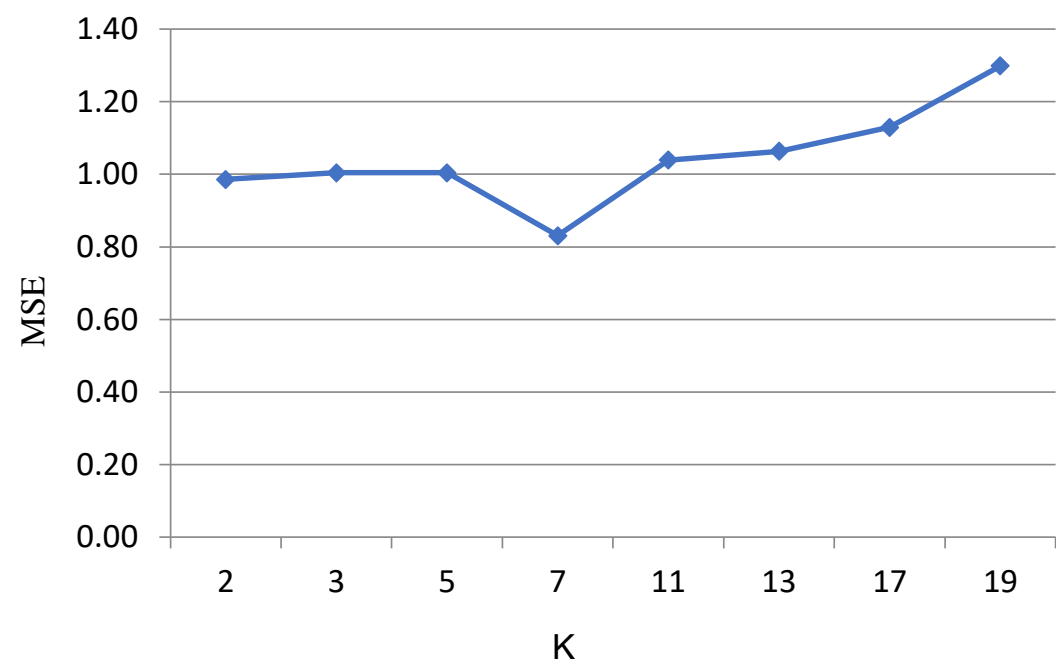

Figure 9. Mean Square Error (MSE) for Various Values of $K$ using 4 APs

\subsection{KNN with Increased Number of Access Points}

The second model had following features:

- Algorithm: K nearest neighbor using Euclidean Distance

- Goal: Finding the nearest position

- Input: Received Signal Strength from 6 access points (RSS vector)

- Output: Estimated nearest position (can be a, b, c or d)

- Testing: $70 \%$ training set $-30 \%$ testing set

The same previous approach was applied and the accuracy rates for $\mathrm{K}=2,3,5,7,11$, $13,17,19$ were $0.742515,0.754491,0.730539,0.778443,0.748503,0.730539,0.694611$ and 0.688623 as shown in Figure 10. The MSEs for $K=2,3,5,7,11,13,17,19$ were $1.052099,0.8690323,0.9880143,0.7810455,0.8819629,0.9820563,1.140272,1.181851$ as shown in Figure 11. 


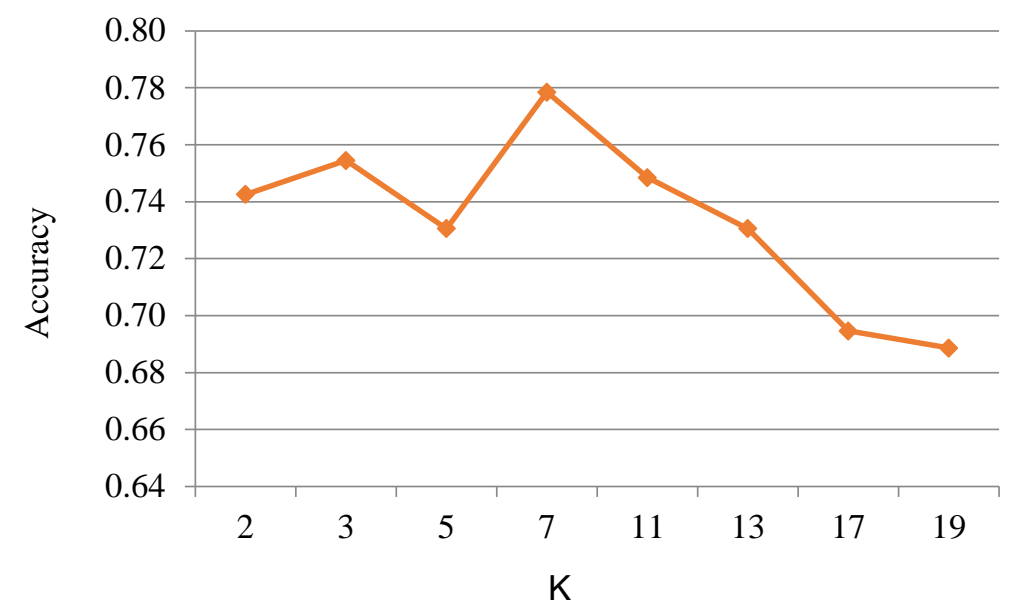

Figure 10. Accuracy Rate for Various Values of K using 6 APs

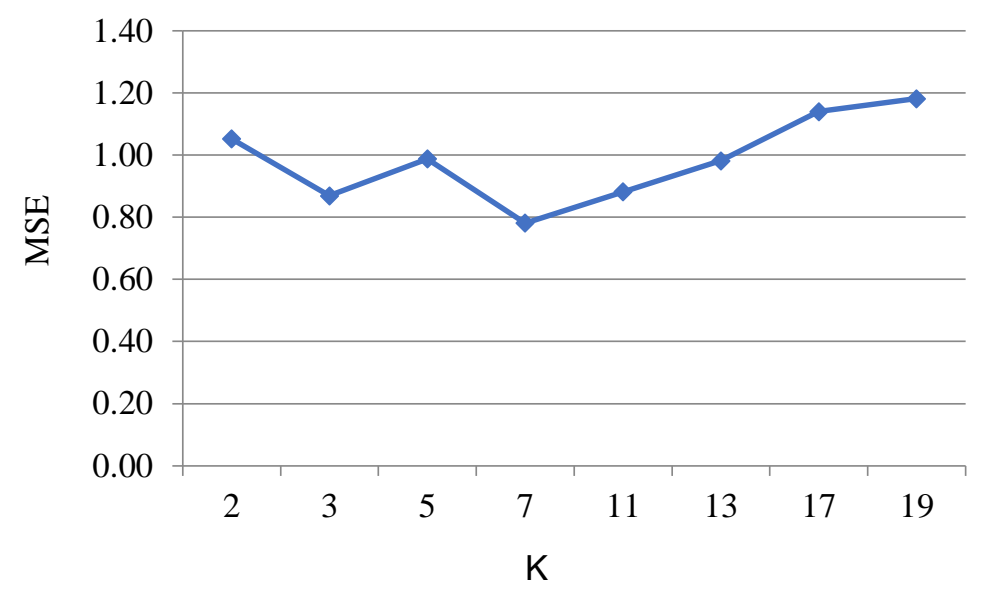

Figure 11. Mean Square Error (MSE) for Various Values of $\mathrm{K}$ using 6 APs

\subsection{Cross Validation for KNN}

For evaluating the result and performance of an algorithm over the dataset, k-fold cross-validation model is used which is a common technique in literature. Especially when we are doing the comparison between models, it is effective to compare them by their performances averaged within a number of iterations of cross-validation. Crossvalidation is a method that is used for assessment of a classifier. In cross-validation model, data set will be divided into two sub sets: training set and test set. In k-fold crossvalidation, the original data set will be divided into $\mathrm{k}$ subsamples partitions randomly. A single subsample is reserved as the validation data for testing usage, and the remaining $\mathrm{k}$ -1 subsamples are used for training. The cross-validation process will be repeated $\mathrm{k}$ times, while each of the $\mathrm{k}$ subsamples used exactly once as the test data. The $\mathrm{k}$ results from the folds are then averaged to produce a single performance estimation [12].

In this project, we applied $\mathrm{k}$-fold cross validation with $\mathrm{k}=10$, and the percentage for correctness of KNN classifier was calculated. The result for model with 4 APs was $69 \%$ and 6 APs was $76 \%$. Figure 12 and 13 show the diagram for k-fold cross validation error rates for KNN model with 4 APs and 6 APs. From these diagrams, it is obvious that by increasing the neighbors, the error increases in both models. But when $\mathrm{K}=1$, we obtained different result in 4APs and 6 APs models, where the error is less in 4 APs compared to 6 APs model. For $\mathrm{K}<3$, the error rate is not reliable with $\mathrm{k}$-fold cross validation, because when we tested the algorithm in both models the MSE value for 4 APs was greater than 6 
APs model, which is contadict and we need to evaluate the accuracy by testing various number of $\mathrm{K}$ in both models.

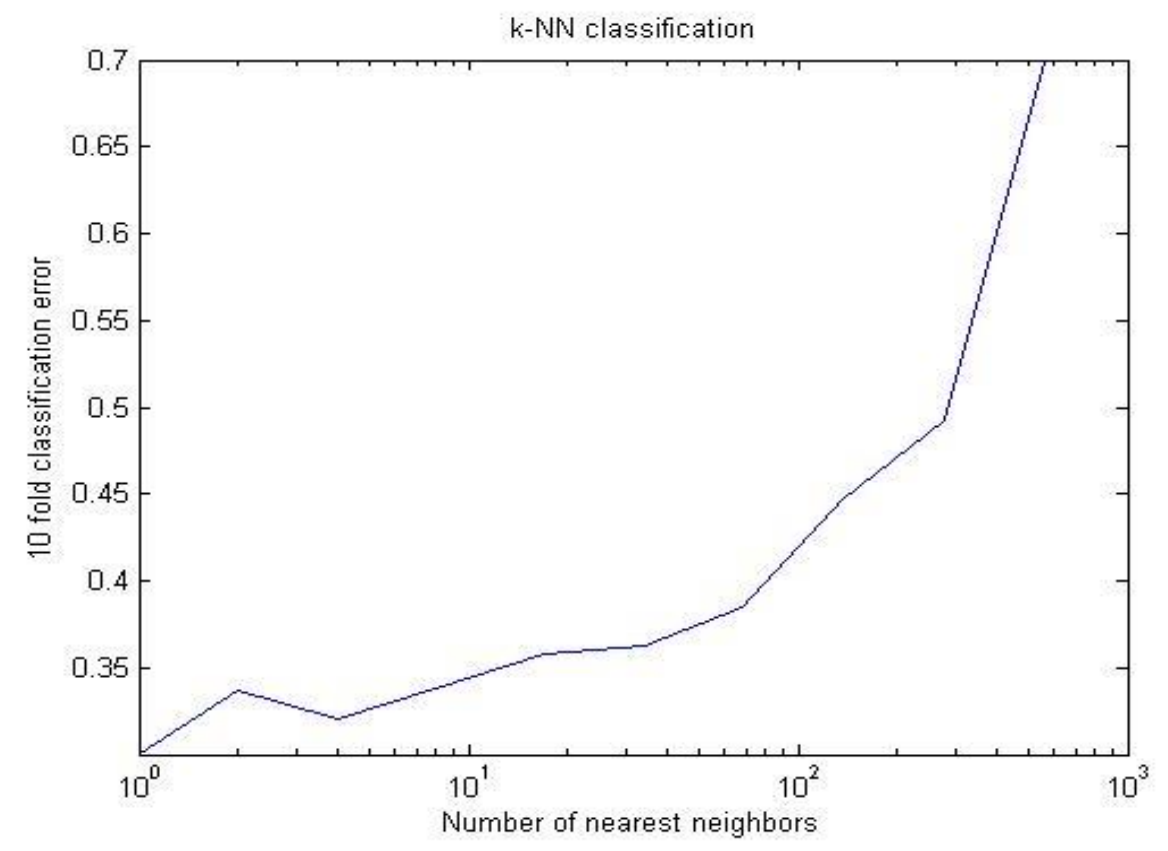

Figure 12. 10-fold Cross Validation for KNN with 4 APs

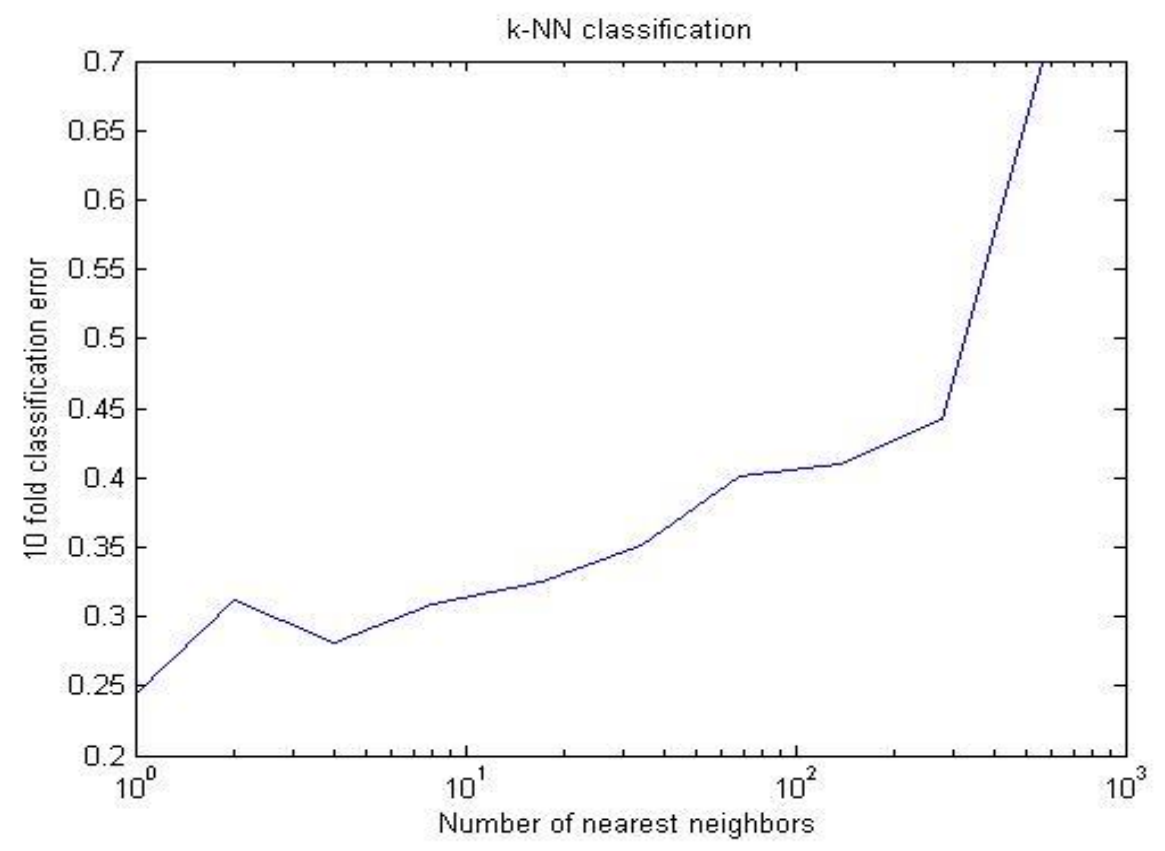

Figure 13. 10-fold Cross Validation for KNN with 6 APs

\section{Result and Discussion}

The KNN approach has been used for positioning in this project. We applied two models for KNN algorithm:

i. Using 4 numbers of access point (AP)

ii. Using 6 numbers of access point (AP) 
The most accurate result for 4 APs model is shown in Table 2.

Table 2. Most Accurate Result for KNN using 4 APs

\begin{tabular}{|c|c|c|}
\hline $\mathbf{K}$ & Accuracy & MSE \\
\hline 3 & $71 \%$ & $0.98 \mathrm{~m}$ \\
\hline 7 & $74 \%$ & $0.83 \mathrm{~m}$ \\
\hline 11 & $72 \%$ & $1.03 \mathrm{~m}$ \\
\hline
\end{tabular}

According to the result in Table 2, we found that by increasing the value of $\mathrm{K}$, the accuracy initially increasing and reduced after specific value of $\mathrm{K}$. Moreover, value of MSE is changed accordingly, the error value by increasing $\mathrm{K}$ was reduced initially, then it increased after a specific value of K. While accuracy increases, MSE value is decreasing, with the least distance error, and it is considered as the best value for accuracy. So, it has been observed that, the best accurate result is at $\mathrm{K}=7$ because we have the least MSE value and the highest accuracy rate.

Next, we increased the number of access point to 6 APs and Table 3 shows the most accurate result for this model.

Table 3. Most Accurate Result for KNN using 6 APs

\begin{tabular}{|c|c|c|}
\hline $\mathbf{K}$ & Accuracy & MSE \\
\hline 3 & $75 \%$ & $0.86 \mathrm{~m}$ \\
\hline 7 & $77 \%$ & $0.78 \mathrm{~m}$ \\
\hline 11 & $74 \%$ & $0.88 \mathrm{~m}$ \\
\hline
\end{tabular}

After increasing the number of APs, most RSS data was fed to the model that gave higher accuracy with reduced MSE value. But the interesting point is that the fluctuation of result is similar to 4 APs model. According to Mok and Cheung [13], by increasing APs, the accuracy increased, since the number of inputs is increased so we can get better accuracy with increasing value of MSE compared to 4 APs model. This is because by increasing the input data, more noises included, thus produces more error. Same as the previous model, the highest accurate rate was achieved when $\mathrm{K}=7$ with the least MSE value. In comparison with Mok and Cheung [13] model, this model achieved higher accuracy.

For 4 APs model, the most precise result occurred when $\mathrm{K}=7$ and $\mathrm{K}=11$ with $74 \%$ and $72 \%$ accuracy respectively. However, in 6 APs model, the most accurate result was at $\mathrm{K}=7$ and $\mathrm{K}=3$ with $77 \%$ and $75 \%$ of accuracy respectively as shown in Table 4.

Table 4. The Most Precise Result of KNN Algorithm

\begin{tabular}{|c|c|c|}
\hline Features & K & Accuracy \\
\hline \multirow{2}{*}{4 APs } & 7 & $74 \%$ \\
\cline { 2 - 3 } & 11 & $72 \%$ \\
\hline \multirow{2}{*}{6 APs } & 7 & $77 \%$ \\
\cline { 2 - 3 } & 3 & $75 \%$ \\
\hline
\end{tabular}

The result shows that while the number of access points increases, the more input data involve and higher accurate rate is obtained in comparison with 4 access points. However, by increasing the number of $\mathrm{K}$ in both models, the MSE will increase since more points produce more noises, which results more error. The models were tested for various numbers of $\mathrm{K}$ as shown in Figure 14. The best accuracy occurred when $\mathrm{K}=7$ for both models, and reduced as $\mathrm{K}$ increases. 


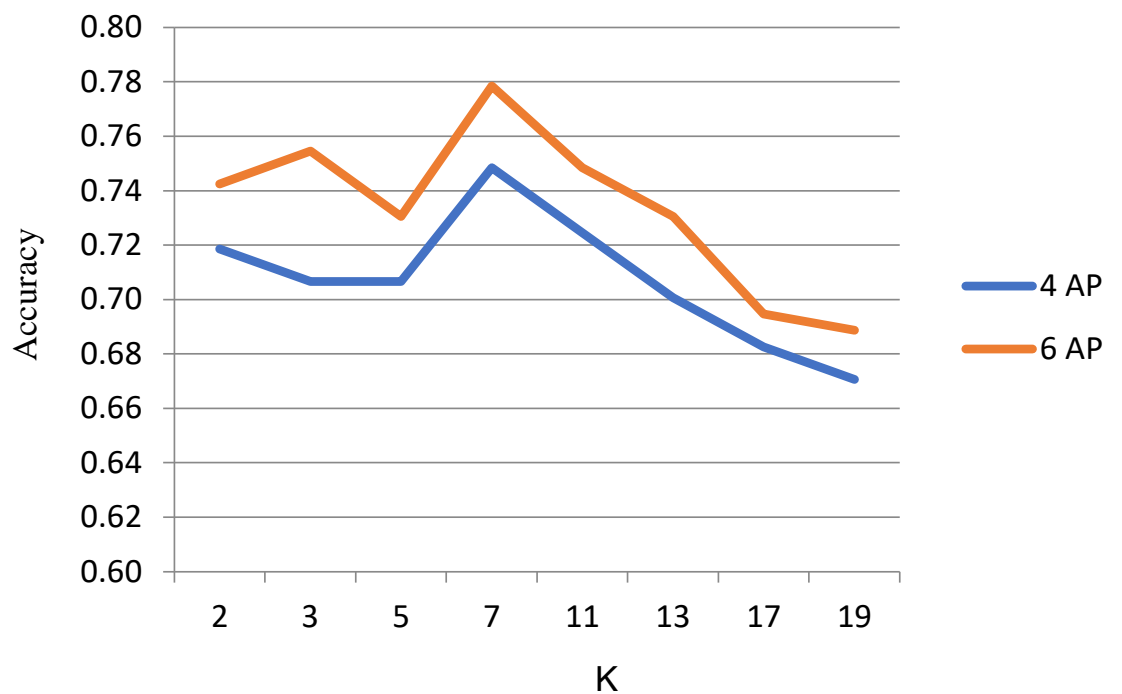

Figure 14. Accuracy Rate for KNN Models

These findings show that KNN has better accuracy with increased number of access points and the more precise result gained in KNN model with 6 APs.

\section{Conclusion}

In each experiment, increasing the number of access point has a goof effect on accuracy and we obtained better result by increasing the number of APs, however, MSE values increase with $\mathrm{K}$. We used one the most commonly used and effective fingerprinting algorithm which most advanced researchers used in their studies. Our approaches were designed in different features and the result were compared and analyzed in many aspects to show the performance. The fingerprinting algorithm used in this study, does not need the coordinates of Wi-Fi access points in the positioning. So this method is convenient for Wi-Fi based indoor positioning system for areas such as shopping malls where access point's positions are not possible to be determined precisely. We also applied additional features to our model in order to enhance the accuracy. These features can be used without using additional infrastructure and it can be used from existing Wi-Fi access point in the building. By increasing the numbers of access points for input data, we reduced the error value. In both models, by increasing the value of $\mathrm{K}$, more data involves in positioning and accordingly more higher error values. However, by employing more access points, signal strengths improved the accuracy to get more precise results with less MSE value.

\section{Acknowledgement}

We would like to express our gratitude to Ministry of Higher Education (MOHE Malaysia) for providing financial support (research grant Q.K130000.2538.11H85) in conducting our study. Our special thanks to Universiti Teknologi Malaysia (UTM) and specifically Advanced Informatics School (AIS) for realizing and supporting this research work.

\section{References}

[1] M. Jain, R. C. P. Rahul and S. Tolety. "A Study on Indoor Navigation Techniques using Smartphones, International Conference on Advances in Computing, Communications and Informatics (ICACCI), (2013).

[2] A. Belakbir, M. Amghar and N. El Kouhen, "An Indoor Positionning System based on the Fusion of 
Location Information", Colloquium in Information Science and Technology (CIST), (2012), pp. 32-37.

[3] Y. Xu and Y. Sun, "Neural Network-Based Accuracy Enhancement Method for WLAN Indoor Positioning", IEEE Vehicular Technology Conference (VTC Fall), (2012).

[4] H. Bao and W.-C. Wong, "An Indoor Dead-Reckoning Algorithm with Map Matching", 9th International Wireless Communications and Mobile Computing Conference (IWCMC), (2013).

[5] H. Vathsangam, A. Tulsyan and G. Sukhatme, "A Data-Driven Movement Model for Single CellphoneBased Indoor Positioning”, International Conference on Body Sensor Networks (BSN), (2011).

[6] S.-E. Kim, Y. Kim, J. Yoon and E. S. Kim, "Indoor positioning system using geomagnetic anomalies for smartphones", International Conference on Indoor Positioning and Indoor Navigation (IPIN), (2012).

[7] S. Jeon, J. Lee, H. Hong, S. Shin and H. Lee, "Indoor WPS/PDR Performance Enhancement Using Map Matching Algorithm with Mobile Phone", IEEE/ION Position, Location and Navigation Symposium PLANS, (2014).

[8] J. L. Liu, Y. H. Wan, B. G. Xu, S. L. Tang, X. K. Ding and Q. Wan, "A Novel Indoor Positioning Method Based on Location Fingerprinting", International Conference on Communications, Circuits and Systems (ICCCAS). (2013).

[9] Z. Xu, R. Wang, F. Li and Y. Huang, "AENS: Accurate and Efficient Mobile Phone Indoor Navigation System without WiFi", IEEE $11^{\text {th }}$ International Conference on Dependable, Autonomic and Secure Computing (DASC), (2013).

[10] B.-J. Shin, K.-W. Lee, S.-H. Choi, J.-Y. Kim, W. J. Lee and H. S. Kim, "Indoor WiFi Positioning System for Android-Based Smartphone", International Conference on Information and Communication Technology Convergence (ICTC), (2010).

[11] Z. Zhu and A. K. Nandi, "Automatic Modulation Classification: Principles, Algorithms and Applications”, John Wiley \& Sons, (2014).

[12] J. G. Moreno-Torres, J. A. Saez and F. Herrera, "Study on the Impact of Partition-Induced Dataset Shift on \&lt;formulaformulatype='inline'\&gt;\&lt;texNotation='TeX'\&gt;\$k\$1t;/tex\&gt;\&lt;/for mula\&gt;Fold Cross-Validation”, IEEE Transactions on Neural Networks and Learning Systems, vol. 23, no. 8, (2012), pp. 1304-1312.

[13] E. Mok and B. Cheung, "An Improved Neural Network Training Algorithm for Wi-Fi Fingerprinting Positioning”, ISPRS International Journal of Geo-Information, vol. 2, no. 3, (2013), pp. 854-868.

\section{Authors}

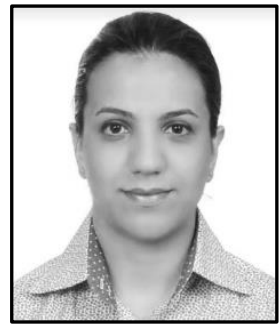

Samaneh Amirisoori, she graduated in Software Engineering and received her B.Sc. from IAU, Tehran, IRAN and received the M.Sc. degree in Computer System Engineering from University Technology Malaysia (UTM), Kuala Lumpur, Malaysia in 2015 and she has received Best Student Awards from UTM University. Her current field placement is with Canadian Imperial Bank of Commerce (CIBC), Toronto, Canada, where she is application developer in technology department She is interested in programming, location based algorithms and software development process.

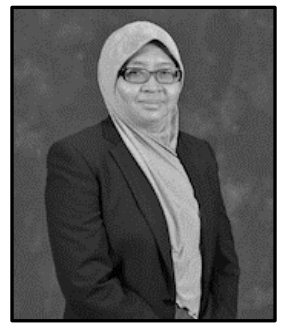

Salwani Mohd Daud, she had completed her Electronics Engineering first degree in University of Liverpool in 1984 and attached to Universiti Teknologi Malaysia (UTM) since $1^{\text {st }}$ November 1985. She had started her career with Faculty of Electrical Engineering (FKE) in 1985, and her teaching experience had exceeded 30 years in UTM. She had obtained her Masters of Electrical Eng. and PhD. (Electrical Eng.) from UTM in 1989 and 2006 respectively. Her main expertise is in machine learning, Internet of Things (Physical layer), security in embedded systems, signal processing and biomedical signal (EEG) processing. Currently she is an Associate Professor in Advanced Informatics School (AIS), UTM Kuala Lumpur. She is the member of Cyber-Physical Systems Research Group. 


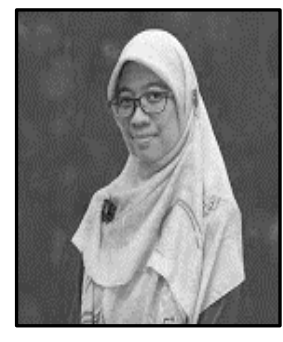

Noor Azurati Ahmad, she serves as a senior lecturer at Advanced Informatics School (UTM AIS), Universiti Teknologi Malaysia Kuala Lumpur. She obtained her B.Eng. in Computer Engineering in 2001 and Master of Electrical Engineering in 2006 from Universiti Teknologi Malaysia. She graduated with a $\mathrm{PhD}$ in Embedded Systems from University of Leicester in 2013. She is a Certified Tester Foundation Level (CTFL) under Malaysian Software Testing Board (MSTB) and Certified Professional for Requirements Engineering (CPRE) under International Requirements Engineering Board (IREB). She has also served as an engineer in Sapura Secured Technologies Sdn. Bhd under the Network Centric Operations (NCO) project. She involved in SIRIM qualification test for Sapura's military products. She is a member of Institute Electricall Electronic Engineer (IEEE), IEEE Computer Society and Registered Graduate Engineer with the Board of Engineers Malaysia (BEM). She has been actively involved in research related to design, software architecture and testing for embedded real-time systems and mobile and pervasive computing.

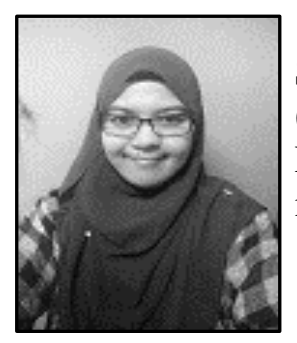

Nur Syazarin Natasha Abd Aziz, she is a Master holder on Science (Computer System Engineering) and Bachelor of Science (Bioinformatics). Currently, she is working at Intel Sdn Bhd as Test Equipment/Process Engineer. She is enthusiastic towards learning new things in across all fields.

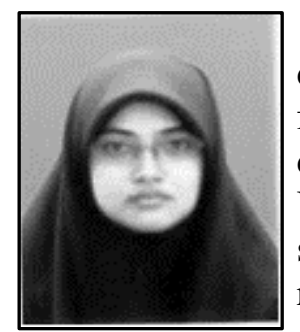

Nurul Iman Mohd Sa'at, she is a research member of IOT devices development lab in University Teknologi Malaysia under the faculty of Advanced Informatics School. She received her bachelor degree in Mechatronics Engineering from International Islamic University Malaysia (IIUM) and currently doing her postgraduate study in Universiti Teknologi Malaysia, Kuala Lumpur Campus. Her research interest is in the field of internet of things(IoT) of device and development layer. She is also interested in developing monitoring system of mobile and ubiquitous based on supervisory and data acquisition.

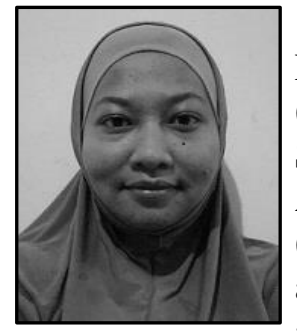

Nur Qamarina Mohd Noor, she received Diploma in Electrical Engineering (Electronics) and B.Eng (Hons) Electrical Engineering (Electronics) from Universiti Teknologi MARA (UiTM), Malaysia. She is currently pursuing MSc in Computer System Engineering in Advanced Informatics School (AIS), Universiti Teknologi Malaysia (UTM). Prior to that, she worked in INTEL (M), Whizz System (M) and MIMOS Berhad in the field of IC failure analysis, RTL design and mixed-signal verification. Her research interest includes hardware security, memory built-in self-test (MBIST), digital and mixed signal verification. 